\title{
ACTITUDES DE LOS ESTUDIANTES DE LA UNIVERSIDAD NACIONAL AGRARIA LA MOLINA HACIA LAS HUMANIDADES
}

María Mandujano Ramos

\section{RESUMEN}

El propósito principal de la investigación fue conocer cuáles son las actitudes de los alumnos de la Universidad Nacional Agraria hacia las humanidades. Este tema tiene importancia en tanto debemos lograr que los estudiantes se apropien de los contextos sociales y culturales que permiten el desarrollo científico, se adueñen de los principios de las materias que estudian. Así, podrán pensar y trabajar de una manera independiente, estar capacitados para comprender los progresos y tener la posibilidad de crear y producir cambios en la ciencia y en la tecnología. En el desarrollo de la presente investigación, se utilizó un cuestionario de actitudes hacia las humanidades, adaptado por la autora del cuestionario formulado por Bazán, J. (1997). Los análisis estadísticos a los que fue sometida la prueba nos indican que el instrumento es válido y confiable. Esta prueba fue aplicada a 386 alumnos de las siete Facultades de la UNALM. Los resultados estadísticos muestran que las actitudes hacia las humanidades se encuentran relacionadas significativamente sólo con la variable estrato social. Por otro lado, es importante señalar que las actitudes hacía las humanidades no son precisamente las mejores, apenas un poco menos del tercio de la población tiene una actitud positiva hacia ella. Con estos resultados sugerimos a la Universidad que no sólo debe implementar cursos y programas específicos, sino incidir en su importancia a fin de formar y capacitar a los estudiantes de modo que puedan estar convenientemente preparados para enfrentar los retos que les impone el mundo actual.

Palabras clave: Actitudes, Humanidades, variables sociodemográficas, Formación profesional, Rendimiento Académico.

\begin{abstract}
The main purpose of the current investigation task was to obtain a description of the attitudes of the students of the Universidad Nacional Agraria La Molina (UNALM) towards the humanities. This topic has the top importance so we have to achieve that the students appropriate of the social and cultural contexts that permit the scientific development, appropriate of the principles of the subjects they study, so they can think and work in an independent way and be capacitated to comprehend the progresses and have the possibility to create and produce changes in the science and technology. In the development of the current investigation, a questionnaire of attitudes towards humanities adapted by the author of the questionnaire formulated by Bazán, J (1997) was used. The statistical analyses the test was submitted to indicate us the instrument is valid and reliable. This test was applied to 386 students of the different faculties of the UNA. The statistical results show us that the attitudes towards humanities are related significantly only with the social stratum variable. It is also important to indicate that the attitudes towards humanities are not precisely the best, only less than the third part of the population has a positive attitude towards her. With these results suggest that the University should not only implement specific courses and programs, but affect their importance to educate and train students so that they can be conveniently prepared to face the challenges arising from today's world.
\end{abstract}

Key words: Attitudes, Humanities, social-demographic variables, professional formation, academic performance.

Revista "Tierra Nuestra" UNALM 


\section{INTRODUCCIÓN}

En pleno siglo XXI, la formación del hombre se encuentra ante el principal reto de su historia: entrenar a las personas para vivir en un mundo cambiante y presidido por la incertidumbre. En este, los individuos han de definir su proyecto vital y estar preparados para habitar un mundo en el que tienen que ser protagonistas. La complejidad de la vida actual plantea al ser humano una amplia gama de problemas y retos que tienen que ver con su posicionamiento en el mundo, con su comprensión de las relaciones sociales y en la ubicación del papel y las responsabilidades que le competen como persona, como educando, a lo largo de su vida y como profesional, en una sociedad cambiante y dinámica.

En ese contexto, se debe reconocer la necesidad de la formación general e integral de nuestros alumnos. Esto debe reflejarse en el resultado final de la formación de los profesionales, quienes no solo deberán conocer su disciplina y ser excelentes técnicos, sino, también, usar un pensamiento crítico para resolver las complejas situaciones en la práctica diaria.

La universidad representa aquel espacio para la búsqueda del conocimiento, la libertad de pensamiento, la excelencia, la posibilidad de crítica, de diálogo dentro de un clima científico de honestidad intelectual. Esta visión de la universidad contextualizada, enfocada y sintonizada dentro del más amplio concepto de desarrollo humano, de plano se contrapone a aquella que solo ve en ella una máquina para producir profesionales.

Desde este punto de vista, el desarrollo humano integral, entendido como la formación de recursos humanos aptos para manejar la ciencia, la tecnología y en general los saberes con criterios éticos, morales y humanistas, consiste en formar al hombre antes que al profesional que cada sociedad requiere. Es por eso que todos nuestros esfuerzos deben encaminarse, en todo momento, a fomentar y consolidar una mentalidad nueva, democrática y profundamente transformadora con respecto a nuestro entorno. Precisamente esta percepción realista del mismo representa el aspecto vital de la denominada educación integral. Nos referimos a ella como a la consolidación de saberes tanto de orden teórico como práctico encaminado al enriquecimiento del mundo espiritual y material de la sociedad; de allí la importancia que tiene la realización de la presente investigación.

El presente estudio contribuirá a aportar datos empíricos sobre un problema que necesita de un nuevo tratamiento que permita adecuarse a las nuevas necesidades que la educación plantea. Además, los resultados nos permitirán diseñar las alternativas que permitan mejorar la formación profesional e integral de nuestros alumnos. Por otro lado, se pondrá a disposición de la comunidad académica y laboral de un nuevo instrumento para evaluar las actitudes de los alumnos hacia los cursos de humanidades. Este instrumento contará con la necesaria validez y confiabilidad para ser utilizado.

En ese sentido, los objetivos fueron describir el nivel de actitudes (afectividad, aplicabilidad, habilidad y ansiedad) que presentan los estudiantes de la Universidad Nacional Agraria La Molina respecto a los cursos de humanidades y establecer la relación que existe entre las actitudes hacia los cursos de humanidades (afectividad, aplicabilidad, habili- 
dad y ansiedad) y las variables socio demográficos (edad, género, facultad de procedencia, tipo de colegio y estrato económico) en los alumnos de la Universidad Nacional Agraria.

\section{MARCO TEÓRICO CONCEPTUAL}

\section{Conceptualización de Actitud}

La actitud es uno de los conceptos de mayor estudio en la Psicología en tanto ellas constituyen valiosos elementos para la predicción de las conductas de los seres humanos. De hecho, nos movilizamos y actuamos de acuerdo a nuestras actitudes.

Las concepciones sobre el término "actitud" son tan variadas como los autores que la han definido. Álvarez y otros (1982) mencionan a Krech quien considera que "una actitud es un sistema duradero formado por componentes de tipo cognoscitivo, sentimental y reactivo que se prolonga en la consecución de un determinado objetivo". En el mismo documento, plantea la concepción de Thurstone en torno a la actitud como la suma total de inclinaciones, sentimientos, prejuicios o distorsiones, nociones preconcebidas, ideas, temores, amenazas y convicciones de un individuo acerca de cualquier asunto específico (Álvarez y otros, 1982).

Otro autor que se refiere a este tema es Pallarés (1981), quien manifiesta que: "La actitud es una tendencia existente en la personal a actuar de un modo determinado cuando se encuentra ante ciertas personas, hechos o ideas. Estas personas, hechos o ideas que provocan este particular modo de obrar o actuar, son los objetos de la actitud" (Pallarés, 1981).
Por su parte, Hernández (1997) manifiesta que "una actitud es una predisposición aprendida para responder consistentemente de una manera favorable o desfavorable ante un objeto de su símbolo". Otro autor que se refiere a este tema es Kerlinger (año?) quien plantea que: "La actitud es una predisposición organizada para pensar, sentir, percibir y comportarse hacia un referente u objetivo cognitivo. Se trata de una estructura perdurable de creencia que predispone al individuo a comportarse de manera selectiva hacia los referentes de actitud. Un referente es una categoría, una clase o un conjunto de fenómenos: objetos fisicos, eventos, conductas e inclusive constructor" (2001).

Carvajal manifiesta que: "Las actitudes son variables no observables que se relacionan con algún conocimiento sobre el objeto al que se reacciona a favor o en contra y que predispone a la acción. Se infiere de esto que es posible predecir conducta del individuo por el conocimiento de la actitud hacia un objeto" (1993).

Quizás la concepción más popular de la actitud es la que formularon Katz y Stotland (1959, en Summers, 1976). Es una definición que integra a la mayor parte de los conceptos presentados anteriormente, y que posee tres componentes:

a)Cognoscitivo, es decir las creencias que se tienen acerca de un objeto. b) Emocional o afectivo, se refiere a las emociones o sentimientos ligados al objeto de la actitud; Lindzey y Aronson (1969), citado por Dawes (1983), dicen que el componente afectivo tiene que ver con la sensación de agrado o desagrado del objeto de la actitud, c) Tendencia a la acción, que según Penner (1976), 
citado igualmente por Dawes (1983) considera en los sentimientos de una persona sobre cómo ella y los otros deberían actuar hacia el objeto de la actitud y/o su comportamiento real hacia dicho objeto.

Por su parte, el estadounidense Gordon Allport (1962) definió actitud como un estado de disposición nerviosa y mental, organizado mediante la experiencia, que ejerce un influjo dinámico u orientador sobre las respuestas que un individuo da a todos los objetos y situaciones con los que guarda relación. En este sentido, puede considerarse la actitud como cierta forma de motivación socialde carácter, por tanto, secundario, frente a la motivación biológica, de tipo primario que impulsa y orienta la acción hacia determinados objetivos y metas.

En general, podríamos decir que las actitudes son las predisposiciones a responder, de una determinada manera, con reacciones favorables o desfavorables hacia algo. Las integran las opiniones o creencias, los sentimientos y las conductas, factores que, a su vez, se interrelacionan entre sí. Las opiniones son ideas que uno posee sobre un tema y no tienen por qué sustentarse en una información objetiva. Por su parte, los sentimientos son reacciones emocionales que se presentan ante un objeto, sujeto o grupo social. Finalmente, las conductas son tendencias a comportarse según opiniones o sentimientos propios.

Las actitudes orientan los actos si las influencias externas sobre lo que se dice o hace tienen una mínima incidencia. También los orientan si la actitud tiene una relación específica con la conducta, a pesar de lo cual la evidencia confirma que, a veces, el proceso acostumbra a ser inverso y los actos no se corresponden, se experimenta una tensión en la que se denomina disonancia cognitiva.

\section{Componentes de las Actitudes}

A partir de lo afirmado por diferentes autores; se podría considerar que los componentes de las actitudes son, fundamentalmente, tres:

Componente cognoscitivo: Para que exista una actitud, es necesario que exista también una representación cognoscitiva del objeto. Está formado por las percepciones y creencias hacia un objeto, así como por la información que tenemos sobre un objeto. Los objetos no conocidos o sobre los que no se posee información no pueden generar actitudes. La representación cognoscitiva puede ser vaga o errónea, en el primer caso el afecto relacionado con el objeto tendrá a ser poco intenso; cuando sea erróneo no afectará para nada a la intensidad del afecto.

Componente afectivo: es el sentimiento a favor o en contra de un objeto social. Es el componente más característico de las actitudes según Rodríguez (1991). Por esto mismo, las actitudes son difíciles de modificar si sólo se emplean métodos racionales basados en el conocimiento objetivo de las cosas; un vínculo afectivo con el alumno (entendido dentro de los parámetros normales de la relación profesor / alumno) puede ser mucho más beneficioso para la modificación de una actitud negativa o perniciosa para el estudio o las tareas encomendadas.

Componente conductual: es la tendencia a reaccionar hacia los objetos de una determinada manera. Es el componente activo de la actitud. Puede ser muy importante en actitu- 
des de negatividad o marginalidad social, generadoras potenciales de actitudes violentas. Frenar el plano conativo de la actitud estimulando el plano emocional suele ser la técnica más usada para el tratamiento escolar de este tipo de casos, y conseguir una cierta modificación. Modificado el plano emocional, se tiene mejor acceso a los planos cognitivo y conativo.

\section{Actitudes hacia las Humanidades}

Se define la actitud hacia las humanidades como el fenómeno que involucra sentimientos (componente afectivo), creencias (componente cognitivo) y las tendencias de los alumnos a actuar de manera particular, acercándose o alejándose del objeto humanidades (componente comportamental). En dicha actitud se hallan contenidos varios aspectos que definen dimensiones tales como: dimensión afectividad (1), que refleja el agrado o desagrado hacia los cursos de humanidades; dimensión aplicabilidad (2), que refleja la valoración a los cursos de humanidades; dimensión habilidad (3), que refleja la confianza en la propia habilidad humanística y dimensión ansiedad (4) que refleja las reacciones comportamentales de ansiedad frente a los cursos.

\section{Dimensión Afectividad}

La expresión afectividad designa una fenomenología tanto personal o endógena como relacional o exógeno. En el terreno personal, constituyen un aspecto fundamental de la vida psíquica del individuo que junto a la inteligencia racional, se identifican con las funciones más importantes en el comportamiento. Del vínculo cognición-afecto se desprende que ambas son esferas interactivas, ya que el ser humano no admite interpretaciones sectoriales sino que todas las funciones internas generan un proceso evolutivo integrado, equilibrado e interfuncional (sensorioperceptual, memoria, pensamiento, lenguaje, cognición, afecto, etc.), hacia la unidad de la personal que motive una "tonalidad afectiva" estable entre emociones y sentimientos.

La manifestación afectiva es vital para un desarrollo psicosocial normal del sujeto. Es, al mismo tiempo, un signo armónico del bienestar emocional y social de la persona. Por ello, creemos que cualquier modelo educativo que lo soslaye será siempre un modelo segregador o segregacionista. Es necesario que las instituciones educativas se ocupen de favorecer el campo de las inteligencias personales, tanto intrapersonales como interpersonales y sociales, incluyéndolas de forma explícita en el currículo, pues potencian el conocimiento afectivo emocional personal y social, facilitándoles la transferencia a su vida relacional de forma activa y eficaz.

Finalmente, entendemos que el vínculo afectivo es una necesidad primaria significativa que establece el nexo entre el individuo y su grupo social de referencia, y solo se puede satisfacer en sociedad. De este modo, alcanzar un desarrollo afectivo pleno, nos introduce en el plano relacional del clima afectivo, entendiendo que éste es la base a partir de la cual se forman las relaciones interhumanas y los lazos que unen al individuo con su medio social (Gutiérrez, 2004).

\section{Dimensión Aplicabilidad}

Constantemente estamos oyendo que el Estado debe proporcionar a la sociedad los que la 
sociedad exige. "¿Qué queremos? ¿La investigación que desea el investigador o la que demanda la sociedad?" La respuesta a la pregunta retórica es obvia: la que demanda la sociedad. Ahora bien, la sociedad demanda lo que conoce, y si no se informa a la sociedad de las ventajas sociales que aporta esa parte de la investigación desvinculada, según se dice, del llamado "bienestar social", es muy difícil que la sociedad lo demande.

Ese es el núcleo de la cuestión: la ciencia es útil; las Humanidades no son ciencia, ergo, las Humanidades son inútiles. Este es un silogismo simple que cumple todos los requisitos exigibles para ser válido y éste en el mensaje que se está lanzando días tras días a la "sociedad". La existencia de las Humanidades se justifica exclusivamente en función de su valor como transmisora de cultura. Algo se puede deducir del énfasis puesto sobre ciertas cuestiones: saber un idioma extranjero (preferentemente inglés) y entender bien un texto. Lo primero puede hacerse en una Escuela de Idiomas, lo segundo debe lograrse en el colegio. ¿Qué nos queda, entonces, a las Humanidades? El destino es claro: la docencia.

Curiosamente, ente la valoración que se da a la Humanidad y la que se otorga a los docentes existe una correlación perfecta. Aquí no hay paradojas. Si las Humanidades son inútiles, es evidente que la valoración de los docentes no puede ser muy alta. De hecho, no lo es. Es por eso que en las universidades de corte claramente técnico, los cursos de humanidades tienden a desaparecer, olvidándose la importancia que tiene la cultura para el proceso de formación profesional del estudiante y el desempeño profesional futuro.

\section{Dimensión Habilidad}

El desarrollo de una habilidad implica la participación activa del sujeto; que en este caso son los alumnos. Los autores coinciden en que las habilidades son sistemas de actividades tanto psíquicas como prácticas; por tanto una habilidad es el resultado de un entrenamiento para cumplir ciertos objetivos planteados, y mientras ese entrenamiento sea continúo la habilidad será perfeccionada para cumplir con tareas cada vez más eficaces.

Este desarrollo tiene como condiciones necesarias: el esfuerzo práctico, la preparación constante, el entrenamiento y la ejercitación continua; para ello, se debe otorgar especial interés en la previa planeación de la actividad que en su estructuración contemple la secuencia de acciones encaminadas al logro de los objetivos que se plantean en la enseñanza. Sin duda el desarrollo de una habilidad requiere de la apertura de condiciones favorables ; el docente desempeña un importante papel en este proceso.

Es claro, entonces, que una habilidad tiene antecedentes totalmente activos e intencionales, su formación y desarrollo están sujetos a las distintas actividades y operaciones bajo un objetivo y de las condiciones que se propicien para lograr niveles cada vez más altos de la habilidad. Por decir, para que un alumno desarrolle la habilidad de describir se tiene que enfrentar verdaderamente a describir y lo tendrá que hacer tantas veces sea necesario hasta ir logrando mejoras en su descripciones. La mediación docente estará sujeta a las necesidades de ayuda que el alumno requiera a través de los momentos de ejecución, orientación y control de la clase. En 
la tarea de desarrollar habilidades, es también imprescindible realizar en todo momento actividad práctica, cognoscitiva $\mathrm{y}$ valorativa.

Desde este punto de vista la habilidad para las humanidades debe suponer que el alumno se ha visto enfrentado sucesivamente a ellas de tal manera que ha logrado manejarla. De esta manera, el bagaje cultural del alumno cada vez es más importante lo cual redundará positivamente en su formación profesional.

\section{Dimensión Ansiedad}

Todas las personas, desde los niños hasta los adultos más mayores, experimentan ansiedades y miedos de vez en cuando. Sentirse ansioso en una situación particularmente incómoda nunca es agradable. Sin embargo, cuando se trata de los niños, experimentar este tipo de sentimientos es normal y necesario. Sentir y lidiar con la ansiedad puede preparar a las personas más jóvenes a hacer frente a experiencias y situaciones retadoras en la vida.

La definición de la ansiedad es "un aprehensión sin una causa aparente". Suele ocurrir cuando no existe una amenaza inmediata a la seguridad o el bienestar de una persona, sin embargo, la amenaza se siente como algo real. La ansiedad hace que una persona quiera escapar de una situación rápidamente. El corazón late con velocidad, el cuerpo puede que empiece a sudar y pronto la persona sentirá "mariposas" en el estómago. Sin embargo, un poco de ansiedad puede ayudar a las personas a mantenerse alerta y concentradas. Por ejemplo, es bastante normal estar un poco nervioso y estresado antes de un examen.
Esto le puede ocurrir a cualquiera. Cierta dosis de ansiedad anticipatoria, de hecho, puede ayudar a poner manos a la obra y a mantener un rendimiento alto mientras se desarrolla el examen. Pero en algunas personas esa ansiedad es sumamente intensa. Se ponen tan nerviosas antes de rendir un examen que su nerviosismo interfiere en su concentración y su rendimiento e inevitablemente los conducen al fracaso.

\section{La Enseñanza de las Humanidades}

Referirse a la Universidad y a los procesos formativos en la "era del conocimiento", implica, realizar una reflexión, sobre qué es el humanismo y cuál es su verdadero impacto en el ámbito universitario. En segundo lugar, responder el interrogante sobre cuál es papel de las humanidades y su impacto en la universidad. En procura de ordenar la discusión, es preciso definir el concepto.

El humanismo representa la visión antropocéntrica, cuya misión es dignificar lo humano. Por eso, todos los humanistas son luchadores por el mejoramiento estructural que contribuya al desarrollo de la persona. También se entiende por humanismo, el propósito de trabajar por la formación de un hombre nuevo, en una sociedad abierta y democrática. Según Guadarrma (1997), no constituye propiamente una corriente filosófica sino una propuesta que sitúa al hombre como valor central de todo lo que existe, y a partir de esa consideración, subordina toda actividad, a propiciarle mejores condiciones de vida material y espiritual.

Humanista o humanismo no es simplemente realizar estudios de latín, griego o de literatura renacentista, y lo que es peor, citar a gran- 
des pensadores o representantes del humanismo como creen algunos intelectuales. Humanista es militar en la causa del hombre, asumiendo todas sus consecuencias políticas.

Santa (1988) al referirse a las cátedras de humanidades plantea: "muchos son los que hoy consideran las humanidades como simples disciplinas intelectuales sin ningún sentido, ni utilidad en la enseñanza universitaria; otros las reducen a simples cátedras cuyo objeto está en suministrar al estudiante datos intrascendentes de cierta cultura general", sin duda, se trata de un reduccionismo conceptual que sólo favorece el menosprecio por las humanidades.

Los aspectos básicos del currículo universitario deben buscar brindar (amontonamiento de verbos) una sólida formación humanística y ética, que garanticen el ejercicio de la formación del nuevo profesional que requiere la sociedad del conocimiento enfrentándolo al modelo pedagógico vigente que tiene una visión eficientista de la enseñanza, que solo habla de calidad, competencia y de pragmatismo.

Se entiende que la relación enseñanzaaprendizaje es un proceso eminentemente humano, sometido a la complejidad de un ser que no puede ser reducido a la mera instrumentalización. El aula, por lo tanto, debe ser un espacio vivo, donde las relaciones intelectuales y afectivas están mediadas por la ética, en el cual solo primará el intercambio comunicativo con actitudes participativas siempre en la búsqueda de colaboración y la superación del conflicto.

Es claro que hoy se requiere de profesionales formados con nuevos criterios de calidad y pertinencia para responder en la sociedad del conocimiento, que influyan considerablemente en el desarrollo del país. Tal pretensión no es posible sin contar con el concurso de las humanidades.

\section{METODOLOGÍA}

Para la selección del diseño de investigación, se ha utilizado como base el libro de Hernández, Fernández y Baptista (2006) titulado "Metodología de la Investigación". Según estos autores, el diseño adecuado para esta investigación es de tipo descriptivo correlacional. Dentro de los diversos tipos de investigaciones convencionalmente aceptadas, la presente línea de investigación, por su finalidad, es básica, ya que su interés fundamental es obtener nuevos conocimientos referidos a las actitudes que presentan los alumnos respecto de los cursos de humanidades.

\section{Población y Muestra}

La población objeto de la investigación está conformada por la totalidad de los alumnos de la Universidad Nacional Agraria La Molina. De la población señalada, se seleccionó una muestra representativa mediante la técnica de muestreo aleatorio y por afijación proporcional. Para la estimación del tamaño de la muestra, se utilizó la siguiente fórmula (Sierra Bravo, 1995).

$$
n=\frac{N^{*} Z^{2} a p^{*} q}{d^{2} *(N-1)+Z^{2} a^{*} p^{*} q}
$$


Tabla $\mathbf{N}^{\circ} 1$

Composición de la muestra por Sexo

\begin{tabular}{ccc}
\hline Sexo & Frecuencia & Porcentaje \\
\hline Hombre & 192 & 49.7 \\
Mujer & 194 & 50.3 \\
Total & 386 & 100.0 \\
\hline
\end{tabular}

Tabla $\mathbf{N}^{\circ} 2$

Composición de la muestra por Facultad

\begin{tabular}{ccc}
\hline Facultad & Frecuencia & Porcentaje \\
\hline Agronomía & 62 & 16.1 \\
Ciencias & 57 & 14.8 \\
Ciencias Forestales & 33 & 8.5 \\
Economía y & 87 & 22.5 \\
Planificación & 36 & 9.3 \\
Industrias Alimentarias & 40 & 10.4 \\
Ingeniería Agrícola & 32 & 8.3 \\
Pesquería & 39 & 10.1 \\
Zootecnia & 386 & 100.0 \\
Total & & \\
\hline
\end{tabular}

Tabla $\mathrm{N}^{\circ} 3$

Composición de la muestra por Colegio de Procedencia

\begin{tabular}{ccc}
\hline Colegio & Frecuencia & Porcentaje \\
\hline Privado & 216 & 56.0 \\
Estatal & 170 & 44.0 \\
Total & 386 & 100.0 \\
\hline
\end{tabular}

Tabla $\mathbf{N}^{\circ} 4$

Composición de la muestra por Estrato Social

\begin{tabular}{ccc}
\hline Estrato & Frecuencia & Porcentaje \\
\hline Bajo & 207 & 53.6 \\
Medio & 104 & 26.9 \\
Alto & 75 & 19.4 \\
Total & 386 & 100.0 \\
\hline
\end{tabular}




\section{RESULTADOS Y DISCUSIÓN}

\section{Resultados descriptivos}

Los resultados observados en la tabla $\mathrm{N}^{\circ} 5$ nos muestran que el $33.7 \%$ de los sujetos de la muestra presentan Actitudes hacia las $\mathrm{Hu}$ manidades en un bajo nivel, mientras que el $34.7 \%$ está en un nivel medio y el $31.6 \%$ está en un nivel alto. Más adelante, detallaremos cuán preocupante es este resultado.

Tabla $N^{\circ} 5$

Análisis de las Actitudes hacia las Humanidades

\begin{tabular}{lcr}
\hline Niveles & Casos & Porcentaje \\
\hline Bajo & 134 & 33.7 \\
Medio & 130 & 34.7 \\
Alto & 122 & 31.6 \\
Total & 386 & 100.0 \\
\hline
\end{tabular}

\section{Análisis correlacionales}

Los resultados presentados en la Tabla $N^{\circ} 6$ indican que existen correlaciones significativas y negativas en el caso de la dimensión ansiedad con la Facultad del alumno y corre- laciones significativas en los casos de afectividad, habilidad, ansiedad y en el total de las actitudes hacia las humanidades con el estrato social de procedencia de los alumnos.

\section{Análisis comparativos}

El análisis comparativo de la Prueba de Actitudes hacia las Humanidades por Sexo (ver Tabla $N^{\circ} 7$ ), demuestra que no existen diferencias estadísticas significativas por lo que podemos concluir que hombres y mujeres tienen las mismas actitudes hacia los cursos de Humanidades.

El análisis comparativo de la Prueba de Actitudes hacia las Humanidades por colegio de procedencia (ver Tabla $\mathrm{N}^{\circ} 8$ ), indica que no existen diferencias estadísticas significativas por lo que podemos concluir que los alumnos, sin importar el colegio de procedencia, tienen las mismas actitudes hacia los cursos de Humanidades.

\section{Discusiones}

Los análisis psicométricos a los que fue sometida la escala de actitudes hacia las humanidades revelan que todos los ítems forman

Tabla $N^{\circ} 6$

Análisis de las Correlaciones entre las actitudes hacía las humanidades y las variables socio demográficos

\begin{tabular}{|c|c|c|c|c|c|}
\hline Variables & Edad & Sexo & Facultad & Colegio & Estrato \\
\hline Afectividad & 0.03 & -0.03 & -0.05 & -0.05 & $0.15^{\star *}$ \\
\hline Aplicabilidad & 0.02 & 0.04 & -0.06 & -0.00 & 0.090 \\
\hline Habilidad & 0.04 & 0.04 & -0.08 & -0.08 & $0.15^{* *}$ \\
\hline Ansiedad & 0.02 & -0.02 & $-0.16^{* *}$ & -0.08 & $0.17^{\star *}$ \\
\hline Total AH & 0.03 & -0.01 & -0.09 & -0.06 & $0.16^{* *}$ \\
\hline
\end{tabular}


Tabla $\mathbf{N}^{\circ} 7$

Prueba Z de comparación de medias de los puntajes de la Prueba de Actitudes hacia las Humanidades por Sexo

\begin{tabular}{lrrrrr}
\hline & \multicolumn{2}{c}{ Varón } & \multicolumn{2}{c}{ Mujer } \\
\hline Variables & \multicolumn{2}{c}{ N=192 } & \multicolumn{2}{c}{$\mathbf{N}=194$} & Z \\
\cline { 2 - 6 } & \multicolumn{1}{c}{ M } & D.E. & \multicolumn{1}{c}{ M } & D.E. \\
\hline Afectividad & 27.23 & 5.85 & 26.84 & 6.36 & 0.62 \\
Aplicabilidad & 28.39 & 5.93 & 28.84 & 6.08 & -0.72 \\
Habilidad & 27.98 & 5.55 & 28.45 & 5.85 & -0.80 \\
Ansiedad & 25.00 & 4.78 & 24.85 & 4.58 & 0.30 \\
Total AH & 108.61 & 18.13 & 109.00 & 19.80 & -0.19 \\
\hline
\end{tabular}

${ }^{*} p<.05^{* *} p<.01{ }^{* * *} p<.001$

parte de dicho inventario, y corresponden a cada una de las cuatro escalas asignadas previamente. El instrumento es confiable en la medida que sus escalas obtuvieron coeficientes Alfa de Cronbach con valores que oscilan entre 0.71 y 0.82 , obteniendo además un alfa de Cronbach total para toda la prueba de 0.86 .

En cuanto a la validez, el resultado del Análisis Factorial Exploratorio indica que la prueba está conformada por un solo factor que explica el $70.53 \%$ de la varianza total. Alcanza además un valor de 0.77 en la medida de adecuación del muestreo de Kaiser-MeyerOlkin, el mismo que puede considerarse como adecuado. Estos resultados nos indican que la prueba es válida, además de confiable.

En lo que se refiere a los resultados de la investigación, debemos señalar que las correlaciones solo existen entre las actitudes hacia las humanidades y el estrato social. Pareciera ser que las nuevas condiciones del desarrollo obligan a que la formación de los pro- fesionales de la Universidad Nacional Agraria deba ser lo más integral posible.

Estos resultados son preocupantes en la medida de que solamente un tercio de los alumnos tienen actitudes favorables a las humanidades. Debemos considerar que la educación debe sentar sus bases en valores; y no debe ser un simple adiestramiento teórico-práctico. Esto implica que, también en las ciencias, debemos preparar personas reflexivas ante las consecuencias de sus acciones. No solo formar técnicos, sino profesionales con amplios conocimientos científicos relacionados con los valores de la persona. Una medida esencial es no solamente incluir cursos de humanidades en el currículo de las Facultades de la UNALM, sino incidir en su importancia en la formación integral de los estudiantes.

En efecto, las humanidades deben ser consideradas como disciplinas que contribuyen a la formación humana del individuo, por oposición a las disciplinas de carácter científico o técnico. Así, son entendidas como el con- 
junto de ciencias humanísticas que, por su origen y tradición literaria, se distinguen en las facultades universitarias y en la nomenclatura académica, de las ciencias exactas, físicas y naturales.

Estos resultados son congruentes con las propuestas teóricas de Valencia, A. (2006) cuando afirma que "En las universidades donde predomina la ingeniería, los departamentos socio-humanísticos son sólo apéndices, es muy posible que el profesorado no sea de lo mejor, y tales departamentos se mirarán con cierta condescendencia. Por su parte, la mayoría de los profesores de ingeniería de casi todo tipo de universidades, tiende a ser menos que entusiastas sobre el currículo no técnico. Después de todo, la mayoría de ellos nunca recibió una formación humanística adecuada y encaran el asunto con escepticismo".

Por otro lado, los resultados son coincidentes con las propuestas de Bautista-Camino, G. y Flores, L. (2008) quienes afirman que "el problema radica principalmente en establecer los parámetros para llevar a cabo la educación integral de un ingeniero, sin perder el equilibrio entre los conocimientos tecnológicos y humanísticos, además, existe la problemática de lograr que en el estudiante exista un interés no solo por cuestiones técnicas, sino, por prepararse en las áreas que le proporcionen un mejor entendimiento del mundo que lo rodea y así crear en él, una cultura sobre el liderazgo".

Insistimos en el hecho de que las humanidades son fundamentales en la formación y desarrollo del estudiante de ciencias exactas y naturales, sin embargo en la mayoría de los casos son poco valoradas, tanto por los alumnos, como por las instituciones educativas a nivel superior, quienes en los programas de estudio tienden a reducir la matricula de los cursos relacionadas con las humanidades y dar un mayor énfasis en las asignaturas tecnológicas.

\section{CONCLUSIONES}

1. Los análisis correlacionales realizados indican que existen relaciones significativas entre las actitudes hacia los cursos de humanidades (afectividad, habilidad y ansiedad) y la variable estrato social en los alumnos de la Universidad Nacional Agraria. Los descriptivos nos permiten observar que solo un tercio de los alumnos tienen actitudes favorables a las humanidades.

2. El análisis comparativo de la Prueba de Actitudes hacia las Humanidades por Sexo, muestra que no existen diferencias estadísticas significativas, por lo que podemos concluir que hombres y mujeres tienen las mismas actitudes hacia los cursos de Humanidades.

3. El referido al colegio de procedencia, indica que no existen diferencias estadísticas significativas por lo que podemos concluir que los alumnos, sin importar el colegio de procedencia, tienen las mismas actitudes hacia los cursos de Humanidades.

4. El análisis de varianza de un Factor de las Actitudes hacia las Humanidades por Facultad indica que existen diferencias estadísticas significativas en los casos de Aplicabilidad, Habilidad, Ansiedad y el Total de las Actitudes hacía las Humanidades, notándose que en el análisis de las comparaciones por pares a posteriori efectuada a través del test de Scheffé, que los alumnos de la Facul- 
tad de Ciencias Forestales y de la Facultad de Ingeniería Agrícola superaron a los alumnos de las otras Facultades.

\section{BIBLIOGRAFÍA}

ANASTASI, A. (1968). Test Psicológicos. Madrid: Aguilar.

ÁLVAREZ et al. (1982). Diseño y construcción de un instrumento para la evaluación de actitudes hacia el proceso de enseñanza aprendizaje. Costa Rica: Oficina de impresiones.

ALLPORT, G.W. (1962). La naturaleza del prejuicio. Buenos Aires: Eudeba.

BAZÁN, J. (1997). Metodología estadística de construcción de pruebas. Una aplicación al estudio de actitudes hacia la matemática de la UNALM. Tesis para optar el Título de Ingeniero Estadístico. Lima: UNALM

BAZÁN, J y Sotero H. (2000). Una aplicación al estudio de actitudes hacia la Matemática en la UNALM. Anales Científicos de la Universidad Nacional Agraria La Molina. Lima: UNALM.

DAWES, R. (1983). Fundamentos y Técnicas de Medición de actitudes. México, D.F: Limusa.

GUADARRAMA, P. (1997). Humanismo y autenticidad. Madrid: Ed. UNED.

GUTIÉRREZ Moar, M. C. (2004). Afectividad y aprendizaje educativo. Hacia una Pedagogía de la Prevención (Tesis Doctoral). Santiago de Compostela, Universidad de Santiago de Compostela. Facultad de Ciencias de la Edu- cación. (Tesis publicada por la USC en formato CD-ROM con el ISBN 84-9750-325-2).

HERNÁNDEZ, FERNÁNDEZ Y BAPTISTA. (2006). Metodología de la Investigación. México: Mc Graw Hill.

KATZ, D. (1984). El enfoque functional en el estudio de las actitudes. En Estudios básicos en ciencias sociales. (261-298) Barcelona: Hora, S.A

OSSGOOD, D; SUCI, G; TENENBAUM, P. (1986). Medición de Actitudes. México: Trillas.

PALLARÉS, M. (1981). Técnicas e instrumentos de evaluación. Barcelona: CEAC

SANTA, E. (1988). La crisis del humanismo. Bogota: Tercer mundo Editores.

SÁNCHEZ, H., Y REYES, C. (2003). Metodología y Diseños en la Investigación Científica. Lima: Visor

SIEGEL Y CASTELLÁN. (1995). Estadísticas no paramétricas, aplicadas a las ciencias de la conducta. México: Trillas

SIERRA BRAVO, R. (1995). Técnicas de Investigación Social Teoría y ejercicios. Décima edición. Madrid: Paraninfo

SUMMERS, G. (1976). Medición de Actitudes. Mexico: Trillas.

Yi Yi, P. (1989). Actitudes hacia las Matemáticas en una muestra de alumnos de quinto año de secundaria y de sexto grado de primaria del distrito de Jesús María. Memoria de Bachillerato de Psicología. PUCP. Lima: PUCP. 